\title{
Polyporus indigenus I. Araujo \& M. A. Sousa, nova espécie da Amazônia
}

\author{
Izonete de Jesus Araujo Aguiar (') \\ Maria Alves de Sousa $\left(^{2}\right)$
}

\begin{abstract}
Resumo
Uma nova espécie de Polyporus, P. indigenus I. Araujo \& M.A. Sousa, é descrita como nova para a Amazônia Brasileira. Esta nova espécie é muito distinta de suas afins, principalmente, na forma e tamanho do píleo e dos basidiosporos, na falta de zonação, pêlos, escamas e estipe praticamente séssil. Foram investigados sua importância econômica e formas de utilização.
\end{abstract}

\section{INTRODUÇÃo}

C "pão do índio" da Amazônia é amplamente distribuído na região onde encontra condições favoráveis ao seu desenvolvimento, atingindo $60 \mathrm{~kg}$. É conhecido e utilizado como alimento pelo caboclo há vários anos. Inicialmente, tomou-se conhecimento da sua existência stravés de coletas de esclerócios (milita) feitas por leigos em escavações para construção de estradas, casas e arações para lavoura (Maravalhas, 1965).

Engler \& Prant! (1900), Brade (1930), Gonçalves (1937) є Maravalhas (1965) só conheciam o esclerócio deste fungo, atribuindo ser o mesmo igual ao existente no sul do Brasil, identificado como Polyporus saporema Möller. Por este motivo, com exceção de notas (Maravalhas, 1962; Araujo \& Sousa, 1978), nada havia sido publicado sobre o "pão do índio" da Amazônia, em razão da falta de observação dos basidiocarpos.

Este trabalho teve como objetivo verificar se o espécime amazônico era o mesmc identificado como Polyporus saporema, necessitando-se para isto cultura de esclerócios, a fim de obter-se basidiocarpos.
MATERIAL E MÉTODOS

Os esclerócios do "pão do índio", referidos neste trabalho, representam coletas realizadas em 1977 - 1978. Parte do material examinado foi enviado à Divisão de Microbiologia, Alimentação e Nutrição do INPA para análise química.

Após cada coleta do esclerócio foi procedida a descrição macro e microscópica dos espécimes ainda frescos, seguindo-se a tentativa de cultura de pedaços de esclerócio ou de um todo, quando pequeno, deixando-se a parte envolvida pela crusta, fora do solo. Os basidiocarpos fcram observados diariamente e coletados ao atingirem o máximo desenvolvimento, juntamente com o esclerócio de origem. Após a coleta foi realizada a descrição macro e microscópica dos espécimes frescos, seguindo-se a secagem em estufa a $60^{\circ} \mathrm{C}$. Os esclerócios não usados em cultura foram também secados em estufa ou à temperatura ambiente. Como espécimes examinados foram mencionados apenas os esclerócios cultivados.

Nos dados de coleta e no exame macros. cópico foram anotados sempre que possivel o tipo de solo, formação vegetal, forma, peso, consistêrıcia, coloração e dimensões. As medidas indicam comprimento por espessura, sendo dados os valores extremos das medidas encontradas nas coletas efetuadas.

A análise macroscópica do basidiocarpo foi feita a oiho nu e com auxílio de lupa, anotando-se as dimensões, forma, consistência, aspectos da superfície, modo de inserção no esclerócio, disposição dos poros e coloraçăo. A coloração deste, bem como do esclerócio seguiu o "Guide des couleres naturelles" (Locquin, 1975), o qual é aqui abreviado para L.

(1) - Instituto Nacional de Pesquisas da Amazônia, Manaus.

(2) - Universidade Federal da Paraíba, João Pessoa. 
Para exame microscópico do basidiocarpo foram feitas preparações de acordo com Teixeira $(1956,1962)$. As preparações do esclerócic foram realizadas retirando-se fragmentos com auxílio de agulha histológica sob lupa, separadamente, das regióes escuras e esbranquiçadas e, da crusta. As técnicas de coioração usadas foram as propostas por Martin (1924), Kotabla \& Pouzar (1964) e Singer (1975).

Para a contagem linear do número de poros e para as medidas sob lupa, foi usada régua milimetrada. No exame das microestruturas foi utilizado microscópio triocular Kyowa e as medidas foram feitas com a ocular micrometrada Kyowa de fio móvel. Os desenhos foram feitos com auxilio de uma câmara clara Leitz e de um microscópio Zeiss Standard 98 com câmara clara acoplada. Para as fotografias dos poros e da massa interna variegada do esclerócio foi utilizada uma máquina fotográfica ProLaborex, montada sobre lupa estereoscópica triocular Leitz. A terminologia micológica segue à orientação de Fidalgo \& Fidalgo (1967).

O esclerócio recebido pelo $\mathrm{Dr}$. Rolf Singer, em 28-V-1977, proveniente da Fazenda Aruanã,

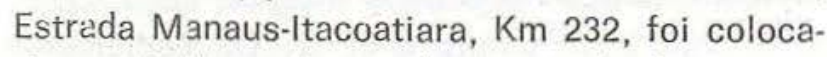
do em balde galvanizado com solo humoso e conservado umedecido no laboratório. Após 2 meses foram observadås pequenas protuberâncias mais ou menos giobosas, conglutinadas ou distanciadas, que se alongaram e se tornaram mais volumosas, determinando conseqüen. temente a concreção de todos os basidiocarpos. Um esclerócio jovem pesando $800 \mathrm{~g}$ coletado, Estrada Manaus-Caracaraí, Km 154 foi plantado em 15-l-1978 em vaso de cerâmica contendo solo humoso e conservado úmido em local sombrio fora do laboratório. Este iniciou sua frutificação no dia 9-VIII-1978. Apresentou crescimento rápido, tendo, após 5 dias, poros nitidamente formados e com 8 dias foi coletado atingindo o diâmetro máximo de $8 \mathrm{~cm}$. De outro esclerócio, coletado no $\mathrm{Km} 8$ da Estrada Manaus-Caracaraí, pesando $2,960 \mathrm{~kg}$, foi plantado um pedaço de $800 \mathrm{~g}$, também em vaso de cerâmica com solo humoso e exposto ao sol, no dia 5-XI-1978 e frutificou em 23-VI-1979, chegando ao máximo desenvolvimento também no período de 8 dias, quando foi coletado. Em todos os experimentos o período de frutifi- cação deste fungo ocorreu entre os meses de junho e agosto; os basidiocarpos originaram-se na região epígea do esclerócio e, mostraram ser efêmeros. Provavelmente, o curto período de írutıficação e duração do basidiocarpo seja a razão do seu desconhecimento na natureza até o presente. Demonstrada a sua época de frutiłıcação, agora, torna-se-á mais fácil a localização do esclerócio, que sendo subterrânєo, antes era encontrado por acaso, através de escavações para construções de estradas, casas e areções para levouras ou mais raramente, quando afloravam.

Após a obtenção e análise dos basidiocarpos, os espécimes amazônicos foram comparados com o tipo de Polyporus saporema Möller conservado no Museu Botânico de Berlim e outros exemplares do Instituto Biológico e Instituto de Botânica de São Paulo, sendo constatado que o "pão do índio" da Amazônia é muito diferente do "pấo do íridio" do su. do Brasil. Desta forma foi dado, neste trabalho, nome à espécie amazônica.

\section{DESCRIÇÃO DA ESPÉCIE}

Polyporus indigenus I. Araujo \& M. A. Sousa, nov. $\mathrm{sp}$.

(Fig. 1 e 2)

Sclerotio perenni, tuberoso, albo (albo, L-H8h), dilute casianeo vel atrocastaneo subrubescenti (russo, L-A2c), crusta tenui, asperata, atroviridi (veneta, L-Z1b), sicca et friabili. Sistemate hiphali dimidico, hyphis generativis et sceleticis usque ad 3-15 $\mu \mathrm{m}$ diametro. Basidincarpo 3,5-5,5 $\times 6-10 \mathrm{~cm}$, ephemero, carnoso spongiosoque; superficie abhymeniali glabra aonataque; sessili vel in pseudostipitem glabrum porosumque affixo. Contexto spongioso flavido (aurantio, L-S4h) usque ad $5 \mathrm{~mm}$ crasso. Hymenophoro tubis unistratificatis, usque 5 $\mathrm{mm}$ longeporis $1-4$ pro $1 \mathrm{~mm}$; dissepimento ad marginem leavi contiguoque. Sistemate hyphali monomitico cum fibulis. Basidio $16,8-22,4 \times$ 5,5-6,7 $\mu \mathrm{m}, 4$-sterigmaticis. Basidiosporis $5,6-7$ x 2,8-3,9 $\mu \mathrm{m}, \pm$ cylindricis, apiculatis inamy. loideisque. Typus in INPA conservatus est. 

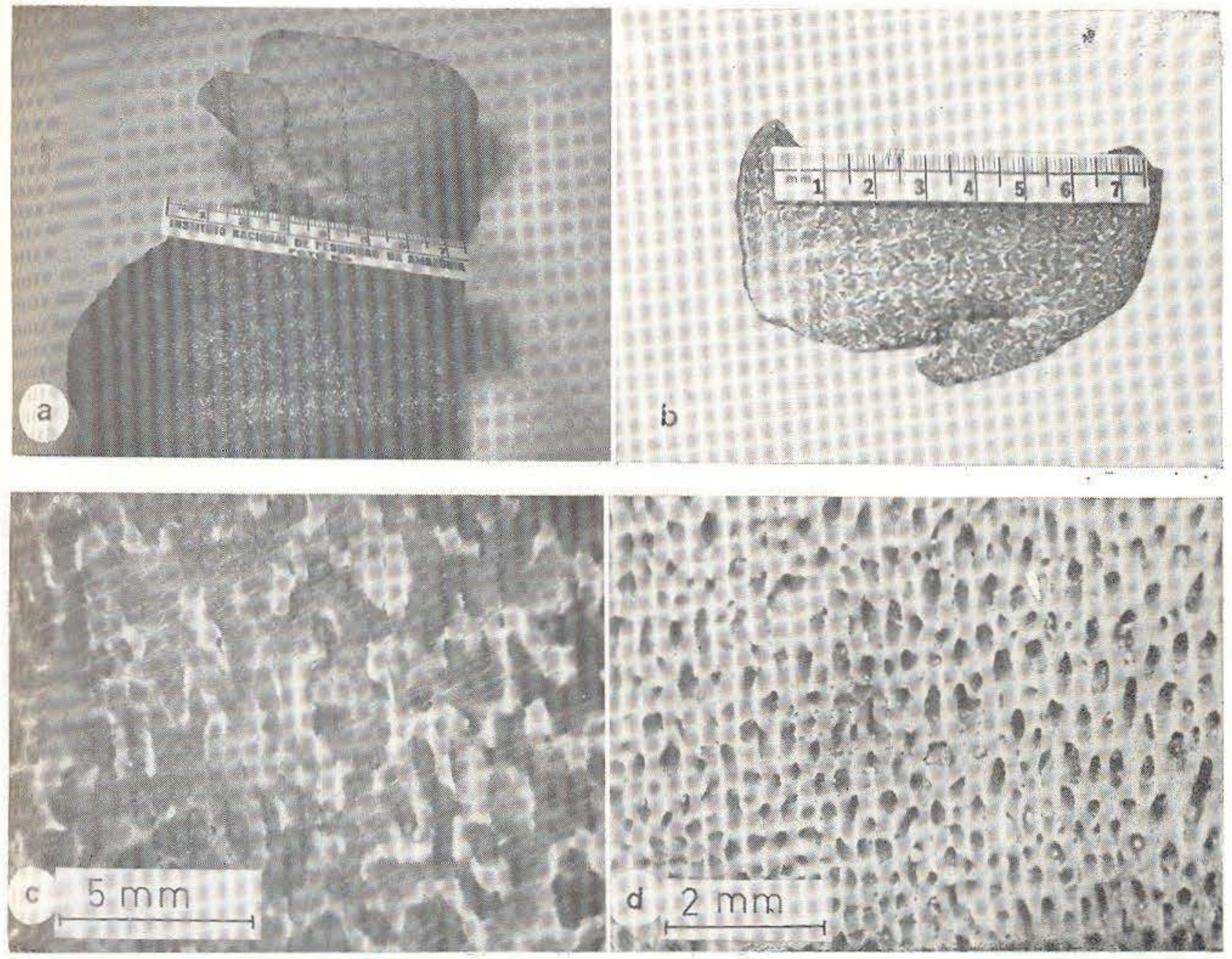

Fig. 1 - Caracteres macroscópicos : a) - Aspecto geral do basidiocarpo no esclerócio; b) — Parte do esclerócio; c) - Massa interna variegada do esclerócio; d) - Poros.

\section{CARACTERES DA ESPÉCIE :}

MACRoscópicos - Esclerócio: perene, muito pesado, tuberoso, $12-50 \times 10-30 \mathrm{~cm}$ nos espécimes examinados, compacto, vítreo opaco, consistência aproximada de borracha, muito duro quando seco, mucilaginoso em $\mathrm{KOH}$, variegado de cores branco (albus, L-H8h) a castanho avermelhado muito claro a escuro (russus, L-A2c); região periférica constituída por uma delgadíssima camada de hifas laxas de cor homogênea laranja (aurantiacus, L-W6h), sobre a qual forma-se uma crusta delgada, áspera, verde escuro (venetus, L-Z1b), seca e quebra. diça mesmo em espécimes frescos, aproximadamente $0,3 \mathrm{~mm}$ de espessura. Basidiocarpo: efêmero, carnoso e esponjoso, solitário ou conglutinado, séssil ou fixo por uma base atenuada formando um pseudo-estipe, forma irre- gular ou aproximadamente circular a dimidiada, 3,5-5,5 $\times$ 6-10 cm; superfície abhimenia! glabra, azonada, convexa, levemente nais compacta que o contexto, amarela (aurantius, L-S4h), e laranja (aurantiacus, LW6h) com a idade; margem espessa, obtusa, glabra, de bordo liso a ligeiramente ondeado. Contexto: esponjoso, amarelo (aurantius, L$\mathrm{S} 4 \mathrm{~h}$ ), até $5 \mathrm{~mm}$ de espessura. Himenóforo: concolor com o contexto ou ligeiramente mais escuro, laranja (aurantiacus, L-W6h), tubos uniestratificados, revestindo toda superfície inferior do píleo até o ponto de inserção no esclerócio, atingindo $5 \mathrm{~mm}$ de comprimento; poros 1-4 por $\mathrm{mm}$, visíveis a olho nu, circulares a oblongos, 115-416 x 92-175 $\mu \mathrm{m}$; dissepimento com margem lisa, glabra, obtusa, 66-222 $\mu \mathrm{m}$ de espessura. 

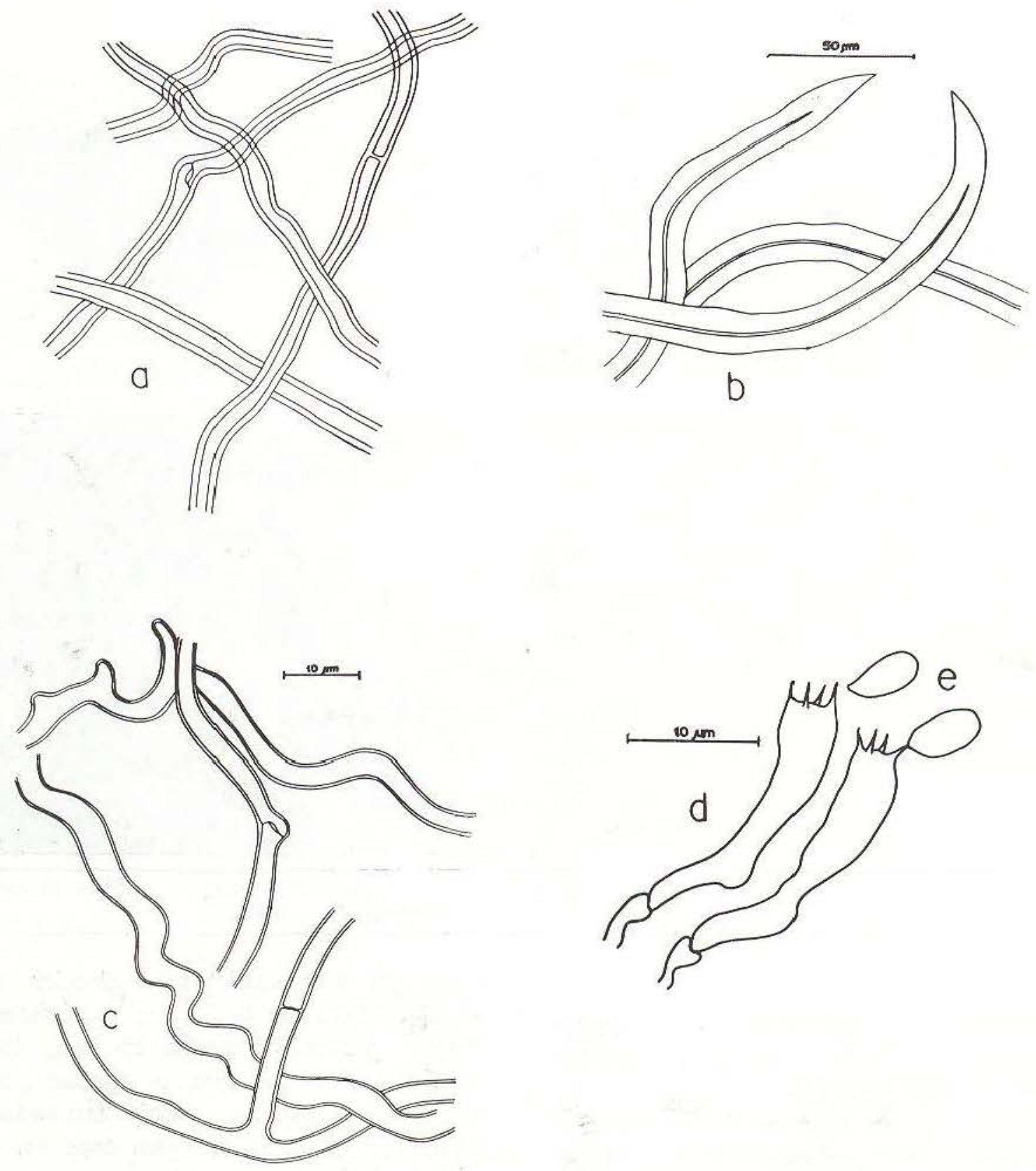

Fig. 2 - a) - Hifas generativas do esclerócio; b) - Hifas .esqueléticas do esclerócio; c) - Hifas generativas do contexto; d) - Basídios; e) - Basidiosporos.

MrCRoscópicos - Esclerócio: sistema hifal dimítico com hifas geradoras e esqueléticas; hifas geradoras hialinas, acianofílicas, algumas apresentam pequenas e abundantes granulações fortemente cianofílicas, paredes delgadas ou ligeiramente espessadas, com fíbulas, septos não muito freqüentes, não ramificadas ou ramificadas, 3-7 $\mu \mathrm{m}$ de diâmetro; hifas es- queléticas hialinas acianofílicas, paredes espessadas e quase sólidas atingindo 1,4-5,9 $\mu \mathrm{m}$, ápices obtusos, em $\mathrm{KOH}$ as exremidades tor. nam-se freqüentemente agudas e curvas e as paredes são mais nitidamente visíveis e de contorno irregular, septos e ramificações não observados, 4,2-15 $\mu \mathrm{m}$ de diâmetro. Basidiocarpo: sistema hifal monomítico; hifas gera- 
doras hialinas, acianofílicas, muitas possuem granulações furtemente cianofílicas, paredes delgadas, com fíbulas, septadas, ramificadas oư não, 2,5-5,9 $\mu \mathrm{m}$ de ciâmetro. Himênio: cistídios e pseudoparáfises ausentes; basídios hialinos, tetra-esterigmados, 16,8-22.4 x 5,5-6,7 $\mu \mathrm{m}$; basidiosporos hialinos, acianofílicos, inamilóides, paredes lisas, apiculados, aproximadamente cilíndricos, 5,6-7 x 2,8-3,9 $\mu \mathrm{m}$.

тIPo: esclerócio, M. Dantas $s / n^{\circ}, 28-\mathrm{V}-1977$ e basidiocarpo, Singer B 9993, 25-VIII-1977 (holótipo - INPA 90190).

LOCALIDADE DO TIPO: Brasil, Amazonas, Estrada Manaus - Itacoatiara, $\mathrm{Km}$ 232, Fazenda Aruanã.

DISTRIBUiçÃo: Brasil - Amazonas, Pará, Rondônia.

eSPÉCIMES EXAMMINAdos: Brasil - Amazonas: Estrada Manaus-Itacoatiara, $\mathrm{Km}$ 232, Fazenda Aruanã, terra firme, capoeira, esclerócio, $M$. Dantas $s / n^{\circ}$, 28-V-1977 e basidiocarpo, Singer B 9993, 25-VIII-1977 (INPA 90190); Estrada Manaus Caracaraí, $\mathrm{Km}$ 154, mata primária de terra firme, I. Araujo \& M. A. Sousa 1379, esclerócio 15-1-1978 e basidiocarpo 16-VIII-1978 (INPA 90191); Estrada Manaus-Caracaraí, Km 8, terra firme, capoeira, M. A. Sousa \& I. Araújo 633, esclerócio 5-XI-1978 e basidiocarpo 30-VI-1979 (INPA 90192).

\section{IMPORTÂNCIA ECONÔMICA}

O esclerócio de Polyporus indigenus, cortado em fatias e frito, é utilizado como alimento pelo habitante da região amazônica, podendo dessa maneira ser também usado como aperitivo. De acordo com nossas experiências, uma das melhores maneiras de ser empregado é no preparo de sopas; para isso, é cortado em pequenos pedacos $\epsilon$ moído, substituindo dessa forma 0 arroz, massas, batatas e outros. O esclerício mesmo fora do solo, pode ser arma. zenado para posterior utilização, pois, em temperatura ambiente, desidrata sem se decompor em um período de 4-6 meses.

Maravalhas (1965) observou que seria possível a uiilizaçáo industrial do esclerócio e fez um estudo preliminar da composição química da polpa, obtendo os seguintes resultados:

$\begin{array}{lr}\text { Umidade } & 49,00 \% \\ \text { Extrato etéreo } & 0,16 \% \\ \text { Cinzas } & 0,18 \% \\ \text { Nitrogenados (N x 6,25) } & 0,175 \% \\ \text { Polissacarídeos e não determinados } & 50,485 \%\end{array}$

A análise do Polyporus indigenus realizada na Divisão de Microbiologia, Alimentação e Nutrição do INPA, forneceu os seguintes dados:

\begin{tabular}{|c|c|c|c|c|c|c|}
\hline & & & & $\%$ & & cal \\
\hline & Umidade & Cinzas & Gorduras & Proteinas & Carboidratos & Energia \\
\hline Polpa & 58 & 0,06 & 1,1 & 0,9 & 39,0 & 173,1 \\
\hline Casca & 35,5 & 1,0 & 1,7 & 2,2 & 59,6 & 262,5 \\
\hline
\end{tabular}

Esses dados demonstram que o "pão do índio" é rico em calorias, revelando que a utilização de $100 \mathrm{~g}$ em uma dieta alimentar proporciona aproximadanente $50 \%$ de calorias necessárias ao organismo humano.

\section{DISCUSSÃo}

Esta espécie possui caracteres peculiares que a distingue de todas as demais portadoras de esclerócio. Maravalhas (1965) referiu-a ccmo sendo Polyporus saporema Möller, espécie mencionada para o sul do Brasil (Engler \& Prantl. 1900). Do espécime da Amazônia anes era conhecido apenas o escierócio. Agora, com a obtenção de basidiocarpos em culturas a partir de esclerócio, foi possível um estudo mais detalhado, demonstrando ser este espécime muito diferente de $P$. saporema. Sendo examinado o basidiocarpo-tipo desta espécie conser- 
vado no Museu Bctânico de Berlim (esclerócio do tipo foi queimado durante a $2^{\mathrm{a}}$ Guerra Mundial), bem como basidiocarpo e esclerócios do Instituto de Botânica de Săo Paulo e Museu Nacional do Rio de Janeiro foram detectados caracteres, sobretudo nos basidiocarpos que os distanciam da espécie amazônica. Além de outros caracteres diferenciais, em $P$. saporema o estipe é mesópode, 3,7-8,5 com de comprimento, castanho acinzentado, densamente piloso com ausência de poros mesmo decurrentes; superfície abhimenial pilosa; tumos curtos com dissepimento freqüentemente descontíguos dando um aspecto irpiciforme, até 2 poros por $\mathrm{mm}$; esclerócio com hifas mais delgadas, 1,5-6,1 $\mu \mathrm{m}$ de diâmetro e basidiosporos maiores, $8,9-13,4 \times 3,2-4,9 \mu \mathrm{m}$. O espécime amazônico é lateralmente séssil por uma base ampla ou pseudo-séssil por uma base aproximadamente mesópoda, atenuada, concolor, glabra e revestida por poros até o ponto de inserção no esclerócio; superfície abhimenial glabra; tubos firmes, mais compridos, com dissepimento contíguos, até 4 poros por $\mathrm{mm}$; esclerócio com hifas mais largas, 3-15 $\mu \mathrm{m}$ de diâmetro e basidiosporos menores, 5,6-7 x 2,8-3,9 $\mu \mathrm{m}$. Segundo Melo (1939) e Tavares (1939) um Polyporus produtor de esclerócio, de Pernambuco, foi determinedo por Pickel como P. pseudosaporema. Entretanto, o tipo desta espécie não foi localizado. De Pleurotus tuber-regium ( Fr) Singer difere, porque apresenta lamelas (Oso, 1977), bem como esclerócio e basidiocarpo dimíticos O "tuchahoe", Poria cocos (Wolf, 1922) que também produz um milita ou paquima muito semelhante ao "pão do índio", chamado "indicus bread", é diferente do amazônico por germinar em laboratório produzindo basidiocarpo ressupinante. Na Austrália, África (Lloyd, 1911) e provavelmente na Índia e Chinâ, ocorrem também esclerócios que produzem frutificações poliporóides férteis, como por exemplo, Polyporus mylittae, espécie australiana, chamada "black fellow's bread" ou "native bread". Todos estes fungos estão relacionados e o "pão do índio" da Amazônia é mais uma espécie nova para o grupo dos fungos produtores de esclerócio,, denominada Polyporus indigenus I. Araujo \& M. A. Sousa, em homenagem ao homem nativo da Amazônia.

\section{AgRADECIMENTOS}

As autoras agradecem ao Dr. William A. Rodrigues pela diagnose latina, ao $\mathrm{Sr}$. Jaime P. L. Aguiar pela análise química, aos curadores dos Herbários $B, R$, SP pelo empréstimo do material para comparação e ao desenhista do INPA, Felipe França Moraes, pela cobertura a nanquim dos desenhos.

\section{SUMMARY}

A new species of Polyporus, P. indigenus I. Araujo \& M.A. Sousa is described. This new species is quite distinct from related species, principally in the size and shape of the pileus and spores, but also in the absence of zonation, hairs or scales and for the stipe being practically sessile. Economic importance and utilization are investigated.

\section{REFERENCIAS BIBLIOGRÁFICAS}

ARAUJO, I. de J. da S. \& SOUSA, M.A.

1978 - Nota prévia sobre o pão do índio da Amazônia Brasileira. Acta Amazonica, 8 (2): 316-318.

BRADE, A.C.

1930 - A saporema. Bol. Mus. Nac., 6 (4): 303305.

ENGLER, A. \& PRANTL, $K$. 1900 - Die Natürlichen Pflanzenfamilien, I: 171-172.

FIDALGO, O. \& FIDALGO, M.E.P.K. 1967 - Dicionário Micológico. Rickia, suplemento 2: $1-232$.

GONÇALVES, R.D.

1937 - Saporema. O Biolćgico, 3 (10): 302-306

KOTLABA, F. \& POUZAR, Z.

1964 - Preliminary results on the staining of spores and other structures of Homodasidiomycetes in cotton blue and its importance for Taxonomy. Feddes Repert., 69 (2): 131-142.

LLOYD, C.G.

1911 - Synopsis of the Section Ovinus of Polyporus. Mycol. Writ., 3: 74-76.

LOCQUIN, M.V.

1975 - Guide des couleures naturelles. De Taxia Fungorum.

MARAVALHAS, N.

1962 - O "Pão de Índio". Ciência e Cultura, 14 (3): 180-181.

1965 - O "Pão de Índio". Ciência e Cultura, 17 (1): $18-20$. 
MARTIN, G.W.

1934 - Three new Heterobasidiomycetes. Mycolo. gia, 26: 261-265.

MELO, P.P.P. de 1939 - O "saporema" de Pernambuco. Bol. da S.A.I.C., 4 (2): 127-129.

OSO, B.A.

1977 - Pleurotus tuber-regium from Nigeria. Mycologia, 69 (2): 271-279.

SINGER, R.

1975 - The Agaricales in Modern Taxonomy. Cramer. 912p.

TAVARES, 1.

1939 - Catálogo dos fungos de Pernambuco. Bol. da S.A.1.C., 4 (1): 1-33.
TEIXEIRA, A.R.

1956 - Método para estudo das hifas do carpóforo de fungos poliporáceos. Publ. Inst. Bot. de São Paulo, 22p.

1962 - Microestruturas do basidiocarpo e sistemática do gênero Fomes (Fries) Kickx. Rickia, 1: $13-93$.

WOLF, F.A.

1922 - In: Weber, G.F. - The ocurrence of Tuckahoes and Poria cocos in Florida. Mycologia, 21 (3): 113-130.

(Aceito para fublicação em 19/02/81) 